\title{
N86- 13799
}

GEOMETRIC-OPTICAL MODELING OF A CONIFER FOREST CANOPY

\author{
Alan H. Strahler \\ Principal Investigator \\ Hunter College \\ City University of New York
}

The primary objective of this research is to explore how the geometry of trees in forest stands influences the reflectance of the forest as imaged from space. Most plant canopy modeling that has been carried out thus far views the canopy as an assemblage of plane-parallel layers on top of a soil surface. For these models, leaf angle distribution, leaf area index, and the angular transmittance and reflectance of leaves are the primary optical and geometric parameters. Such models, and their derivatives, are now sufficiently well developed to explain most of the variance in angular reflectance measurements observed from homogeneous plant canopies. However, forest canopies as imaged by airborne and spaceborne scanners exhikit considerable variance at quite a different scale. Brightness values vary strongly from one pixel to the next primarily as a function of the number of trees they contain. At this scale, the forest canopy is nonuniform and discontinuous.

This research focuses on a discrete-element, geometric-optical view of the forest canopy. Trees are considered to be solid objects casting shadows on a contrasting plane. The brightness of a pixel is then a function of the background brightness, the number of trees in the pixel, the size and shape of the trees and the individual shadows they cast, and the chance overlapping of shadows and tree crowns that occurs when trees fall close together. This model of the forest canopy presents an alternative approach to canopy modeling that is well suited to applications of multispectral imaging by aircraft and spacecraft.

The primary objectives of this research are: (1) mathematical formulation of a discrete-element, geometricoptical model to explain the mean and pixel-to-pixel variance in reflectance of sparse to dense forest canopies: (2) testing of the formulation through Monte Carlo simulations and real image data; and (3) development of an inversion procedure to allow remote estimation of size, shape and spacing of trees from imagery when the imagery is too coarse to permit identification and neasurement of individual trees.

As developed, the model considers conifers as cones (Fig. 1). Paralle1-ray geometry is used to describe the 
illumination of the three-dimensional cone and the shadow it casts on the background. Cones are assumed to be randomly placed and freely overlapping: their heights are assumed to be ognormally distributed. Both these assumptions are supported by literature studies and field work. For the initial model, the cones are assumed to be of a fixed shape determined by the apex angle; however, in application, the apex angle is allowed to vary and an effective apex angle is determined for the stand as a whole.

The research has been carried ou' in four overlapping phases, all of which are nearly complete. The first phase involved development of the basic mathematical model explaining the distribuicion or reflectance values expected for stands with given size, shape, and spacing parameters under fixed conditions of illumination. This phase included the development of an importart general theorem in geometric probability describing the expected variance in random overlapping of shapes in the plane.

The second phase utilized Monte Carlo simulations of forest stands both to confirm the general theorem and empirically calibrate some parts of the model. As one part of the Monte Carlo modeling effort, the bidirectional reflectance of a pixel consisting of Lambertian cones on a background was simulated. The result (Fig. 2) showed many of the features of bidirectional reflectance distribution functions that have been observed for real forests.

The third phase involved assembling Landsat $80-\mathrm{m}$ and SPOT simulation $10-\mathrm{m}$ aigital imagery for two test sites in northern California, and visiting the sites to collect ground data for model calibration and verification. The two sites contrasted a rather open stand of smaller, broader trees (pines) with a dense stand of taller, more narrow trees (red firs). Field measurements included recording the diameter and location of every tree within 12-16 circular subplots arranged on a grid at each site, as well as the collection of accurate heights and apex angles for a random subsample of trees within each subplot.

The fourth phase included the development of an inversion procedure to estimate stand parameters of tree size, shape, and spacing remotely through the observed distribution of brightness values for a stand, as well as the checking of the results against the field observations. For the two stands tested, the inversion procedure yielded good results. The $80 \mathrm{~m}$ imagery was less accurate for the denser of the two stands, whereas the $10-m$ imagery was generally better. The contrasts between the two stands emerged strongly at both resolutions in spite of some differences in observed and calculated parameter values. 
Geometric-optical Modeling

The significance of this research is twofold. First, it establishes firmly that the three-dimensional geometry of ground scenes alone can explain much of the variance in digital imagery seen at resolutions typical of present and planned sensing instruments. This observation is widely applicable beyond forests to many other types of natural plant communities, as well as to any other scenes imaging three-dimensional objects, such as urban areas, suburban developments, etc. Second, it provides a method to directly parameterize the shape, size, and spacing of ground objects remotely even when such objects are below the level of resolution of the sensing instrument. The most obvious example is the remote estimation of forest biomass, which is a direct function of the shape, height, and density of the trees. Used in conjunction with quantitative relationships between spectral reflectance and leaf area index/standing biomass now being developed by other researchers, as well as with a stratified sampling procedure, this technique could greatly aid global understanding of carbon flows and cycles.

The future development of this research will have two main thrusts. First, more mathematical modeling is necessary to incorporate new crown shapes and mixtures of shapes, as well as to account for terrain effects. Second, more field testing is necessary to confirm the utility of the inversion procedure and extend the technique to vegetation types beyond conifer stands. These developments will not only improve our basic understanding of remotely sensed scenes, but also allow the direct extension of that improved understanding to relevant problems in remote sensing and global habitability. 
OF POOR QUALIY

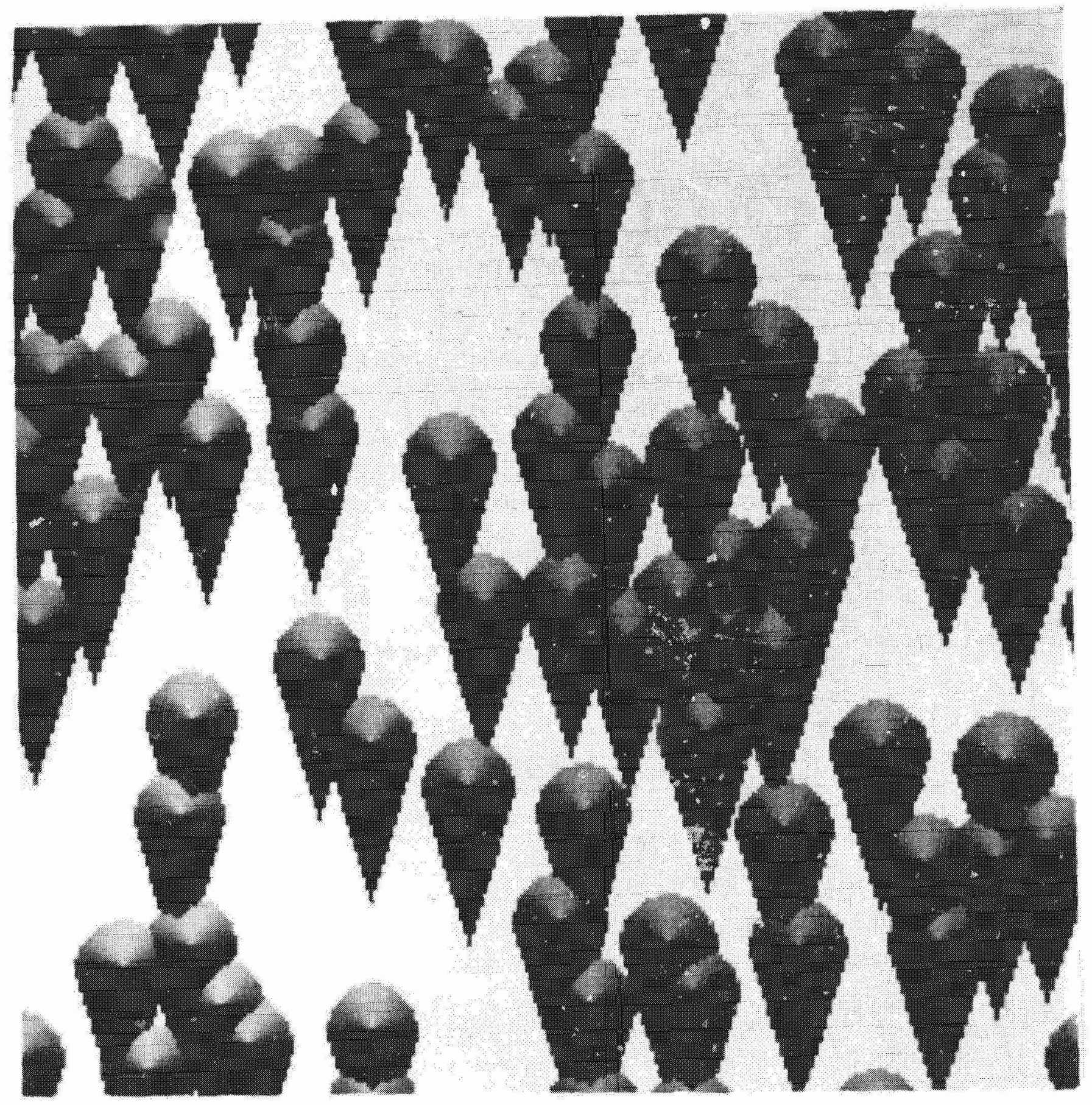




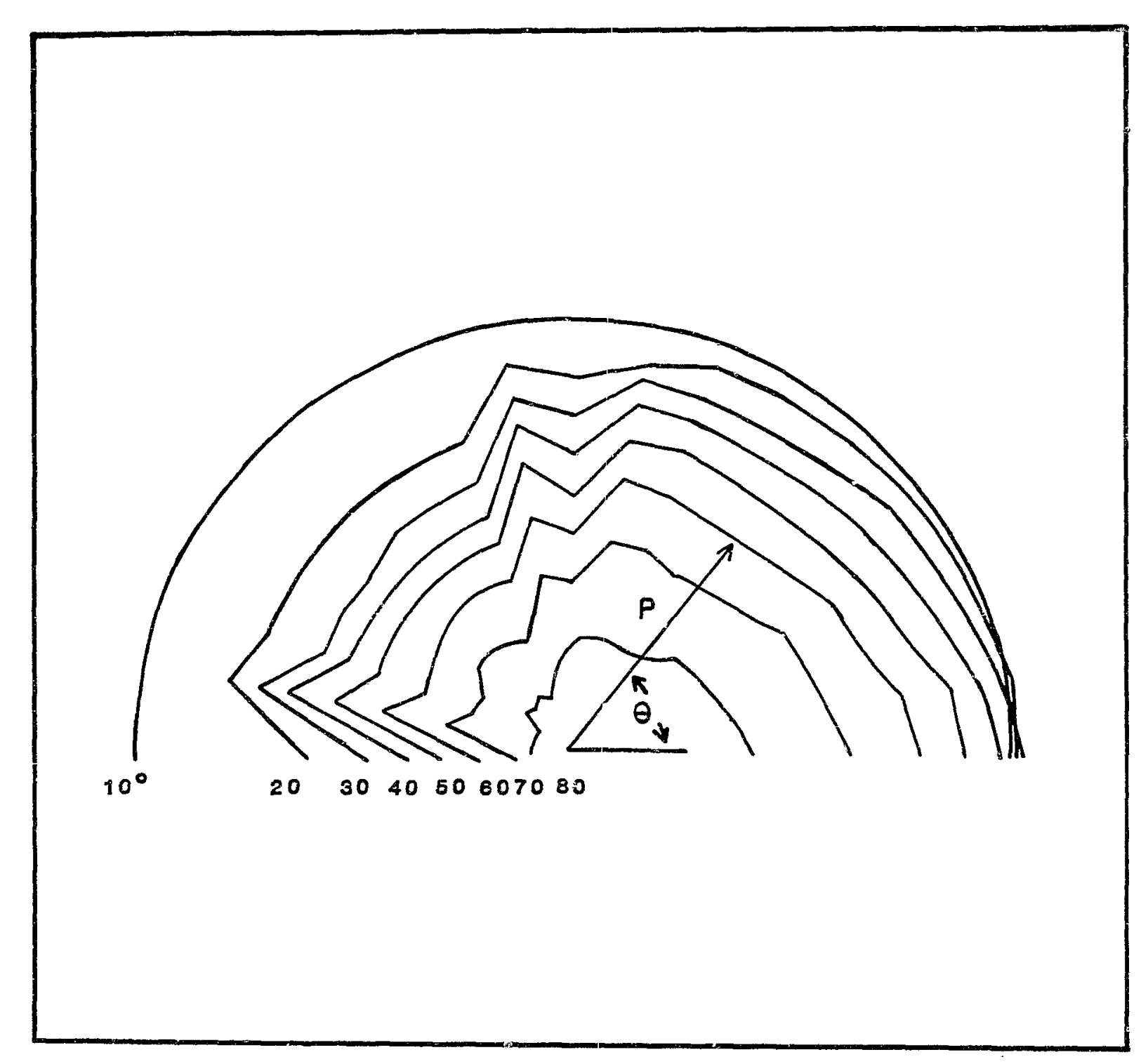

